\title{
Hypoxia promotes migration/invasion and glycolysis in head and neck squamous cell carcinoma via an HIF-1 $\alpha$-MTDH loop
}

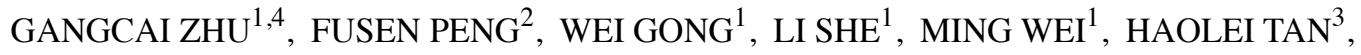 \\ CHANGHAN CHEN ${ }^{1}$, DIEKUO ZHANG ${ }^{1}$, GUO LI $^{1}$, DONGHAI HUANG ${ }^{1}$, \\ $\mathrm{XIN}_{\mathrm{ZHANG}}{ }^{1}$ and YONG LIU ${ }^{1}$ \\ ${ }^{1}$ Department of Otolaryngology Head and Neck Surgery, Xiangya Hospital, Central South University, Changsha, \\ Hunan 410008; ${ }^{2}$ Department of Otolaryngology Head and Neck Surgery, Loudi Central Hospital, \\ Loudi, Hunan $417011 ;{ }^{3}$ Department of Head and Neck Surgery, Hunan Cancer Hospital, \\ The Affiliated Tumour Hospital of Xiangya Medical School, Central South University, \\ Changsha, Hunan 410013, P.R. China
}

Received January 4, 2017; Accepted July 25, 2017

DOI: $10.3892 /$ or.2017.5949

\begin{abstract}
Hypoxia is a hallmark of progressive cancer. Hypoxic cancer cells trigger glycolysis in response to a decreased $\mathrm{O}_{2}$ supply to meet metabolic and bioenergetic demands. Meanwhile, these responses to hypoxia and alterations of the microenvironment promote cancer cell metastasis by increasing transcription of hypoxia-inducible factor (HIF)-regulated genes. However, the detailed mechanism by which hypoxia regulates cancer cell metastasis and glycolysis remains to be investigated. In the present study, we identified that metadherin (MTDH), a multifaceted oncogene, is involved in the regulation of head and neck squamous cell carcinoma (HNSCC) metastasis and invasion under hypoxic conditions. Furthermore, the study indicated that there is a positive feedback loop between HIF-1 $\alpha$ and MTDH in HNSCC cells, and that hypoxia promotes HNSCC cell metastasis and epithelialmesenchymal transition by mediating the HIF-1 $\alpha$-MTDH loop. These findings implicate HIF-1 $\alpha-\mathrm{MTDH}$ as a promising target for anticancer drugs in solid tumors, and help to explain the pro-tumorigenic and unfavorable effect of MTDH on HNSCC observed in our previous studies.
\end{abstract}

Correspondence to: Dr Xin Zhang or Dr Yong Liu, Department of Otolaryngology Head and Neck Surgery, Xiangya Hospital, Central South University, 87 Xiangya Road, Changsha, Hunan 410008, P.R. China

E-mail: xinzhang@csu.edu.cn

E-mail: liuyongent@csu.edu.cn

Present address: ${ }^{4}$ Department of Otolaryngology Head and Neck Surgery, The Second Xiangya Hospital, Central South University, Changsha, Hunan 410010, P.R. China

Key words: head and neck squamous cell carcinoma, MTDH, HIF-1 $\alpha$, hypoxia, glycolysis, metastasis

\section{Introduction}

Head and neck squamous cell carcinoma (HNSCC) affects $\sim 500,000$ new patients annually worldwide (1). HNSCC patients experience vital dysfunctions, including difficulties in breathing, speech and swallowing. Despite advances in the available therapies for HNSCC, including surgery and radio-chemotherapy, the survival time has not significantly improved. Lymph node metastasis is considered to be a major cause of the poor survival times (2). Efforts to improve our understanding of the molecular mechanisms of metastasis may aid in developing novel therapeutic strategies for HNSCC.

Recent evidence has indicated that microenvironmental constituents in the cancer stroma substantially influence the propensity of cancer cells to metastasize (3). Oxygen is a key factor in the microenvironment important for maintaining tissue homeostasis and cellular metabolism by generation of adenosine-5'-triphosphate (ATP). Notably, it has been estimated that regions of hypoxia and/or anoxia account for $\sim 60 \%$ of solid tumors arising as a result of an imbalance between limited oxygen delivery and prolonged consumption (4). Uncontrolled growth of cancer cells results in hypoxia due to the increased distance between the vasculature and certain regions of the tumor. When cancer cells adapt to low oxygen stress, cellular pathways controlling glucose uptake, metabolism, angiogenesis and erythropoiesis are activated by hypoxia-inducible factor-1 $\alpha$ (HIF-1 $\alpha)$ to facilitate cancer cell proliferation and progression (5). Hypoxia, a hallmark of cancer, activates hundreds of transcriptional activities by stabilizing HIF-1 $\alpha$ and inducing its translocation to hypoxia-response elements (HREs) in target genes (6). Hypoxia and HIF-1 $\alpha$ signaling products influence multiple steps within the metastatic cascade, including invasion, migration (Snail, Twist, ZEB1/2, MMPs and CCR5), and establishment of the pre-metastatic niche (LOX, SDF-1, CXCL12, CCL2 and exosomes) (7). In addition, HIF-1 $\alpha$-induced glycolytic gene transcription drives hypoxic cells to produce pyruvate, which is then converted into lactate rather than being oxidized via the tricarboxylic acid (TCA) 
cycle and oxidative phosphorylation (8). This metabolic shift (the Warburg effect) can speed up energy generation, compensating for increased ATP demand and enhancing biosynthesis, allowing tumor cell proliferation and development even in a low-oxygen microenvironment (9). Cancer cell motility and cytoskeleton remodeling have been suggested to be dependent on aerobic glycolysis (10). Previous investigations of tumor cell metabolism have shown that strengthening cancer cell utilization of the glycolytic pathway contributes to cell metastasis (10). Numerous immunochemical studies have indicated that the elevated expression of HIF- $1 \alpha$ and other hypoxiarelated proteins is correlated with lymph node metastasis and poor prognosis in various tumor types, including lung cancer and HNSCC (7).

Metadherin (MTDH), also known as AEG-1 or Lyric, is located on human chromosome $8 \mathrm{q} 22$ and is recognized as an oncogene that regulates numerous signaling pathways in cancer cells (11). Our previous study indicated that high expression of MTDH in HNSCC predicted an unfavorable survival time in HNSCC patients (12), and promoted cell metastasis by inducing angiogenesis and epithelial-mesenchymal transition (EMT) in vitro $(13,14)$. The PI3K/AKT pathway was demonstrated to be involved in MTDH-induced angiogenesis and EMT in our previous studies $(13,14)$. Additionally, HIF-1 $\alpha$ was reported to regulate MTDH expression by activating the PI3K/AKT pathway in glioma cells (15). However, the potential regulatory mechanism associated with MTDH in HNSCC has rarely been investigated. Our preliminary experiments indicated that MTDH expression was elevated in hypoxic cell cultures. However, whether MTDH is involved in hypoxia-induced metastasis and glycolysis in HNSCC cells remains unclear.

In the present study, we confirmed that hypoxia increased MTDH expression via HIF-1 $\alpha$ expression. Knockdown of MTDH expression in HNSCC cell lines interrupted hypoxia-induced metastasis and glycolysis. Furthermore, reduced MTDH expression decreased HIF-1 $\alpha$ expression. The present study indicated that there is a positive feedback loop between MTDH and HIF-1 $\alpha$ in HNSCC. Hypoxia promotes HNSCC metastasis and glycolysis via an MTDH-HIF-1 $\alpha$ loop pathway.

\section{Materials and methods}

Cell line culture and transfection. The Tu686 cell line was provided by Dr Zhuo Chen of Emory University's Winship Cancer Institute in Atlanta, Georgia and was maintained as monolayer cultures in Dulbecco's modified Eagle's medium (DMEM)/F12 medium (1:1) supplemented with $10 \%$ fetal bovine serum (FBS) at $37^{\circ} \mathrm{C}$ under normoxia in a modular incubator chamber, or under hypoxia with $5 \% \mathrm{CO}_{2}$ and $1 \%$ $\mathrm{O}_{2}$ balanced with $\mathrm{N}_{2}$. The medium was changed every other day. Exponentially growing cells were used for the following experiments.

MTDH cDNA (GeneCopoeia, Guangzhou, China), shRNA (sc-77797V; Santa Cruz Biotechnology, Santa Cruz, CA, USA, CA, USA), HIF-1 $\alpha$ cDNA (OriGene Technologies, Inc., Rockville, MD, USA), siRNA (sc-35561; Santa Cruz Biotechnology), and their corresponding control plasmids were transfected into the Tu686 cell line according to the manufacturer's instructions.
Western blotting. All western blot analysis was performed as previously described (16). In brief, loading protein was separated by $10 \%$ sodium dodecyl sulfate-polyacrylamide gel electrophoresis (SDS-PAGE), and transferred onto polyvinylidene difluoride (PVDF) membranes (Millipore, Bedford, MA, USA). The blotted membranes were incubated with the primary antibody against MTDH (1:800; ProteinTech Group, Inc., Chicago, IL, USA), HIF-1 $\alpha$ (1:1,000; Santa Cruz Biotechnology), E-cadherin (1:1,000), N-cadherin (1:800), vimentin (1:800) (all from Cell Signaling Technology, Inc., Danvers, MA, USA) and the secondary antibody $(1: 3,000$; Beyotime, Shanghai, China) in succession. A specific anti- $\beta$ actin protein (1:1,000; Beyotime) was used to detect the quantity of the loading control protein. Each experiment was repeated three times.

Reverse transcription-quantitative polymerase chain reaction (RT-qPCR). Primers for $\beta$-actin, HIF- $1 \alpha$, E-cadherin, vimentin, $\mathrm{N}$-cadherin and VEGF mRNA were synthesized and validated by Beijing Sunbiotech Co., Ltd. (Beijing, China). TRIzol $^{\circledR}$ reagent (cat no. 15596026; Thermo Fisher Scientific, Inc., Waltham, MA, USA) was used for RNA preparation. PowerUp ${ }^{\mathrm{TM}}$ SYBR $^{\circledR}$-Green Master Mix (A25776; Thermo Fisher Scientific) was used in the PCR amplification. The expression level was quantified by an Applied Biosystems device using the $2^{-\Delta \Delta \mathrm{Ct}}$ method. Melting curve analysis was performed at the end of the amplification cycle to verify non-specific amplification. The detailed information of PCR primers is listed as follows: VEGF-L, 5'-aggccagcacata ggagaga-3' and VEGF-A, 5'-tttcttgcgctttcgttttt-3'; HIF-1 $\alpha$-L, 5 '-ccacctatgacetgcttggt-3' and HIF-1 $\alpha-\mathrm{R}, 5$ '-tatccaggctgtgt cgactg-3'; $\beta$-actin-L, 5'-ctcttccagcettccttcct-3' and $\beta$-actin-R, 5'-agcactgtgttggcgtacag-3'; E-cadherin-L, 5'-tgcccagaaaa tgaaaaagg-3' and E-cadherin-R, 5'-gtgtatgtggcaatgcgttc-3'; vimentin-L, 5'-gagaactttgccgttgaagc-3' and vimentin-R, $5^{\prime}$-tcca gcagcttcctgtaggt-3'; N-cadherin-L, 5'-aggatcaaccccatacacca-3' and N-cadherin-R, 5'-tggtttgaccacggtgacta-3'.

Wound healing and Transwell assays. Cells seeded into 6-well plates were allowed to proliferate to near $100 \%$ confluence and were wounded by removing a line of cells using a disinfected Eppendorf tip $(100 \mu \mathrm{l})$. After washing with FBS-free medium $(0 \mathrm{~h})$, the first image was photographed under a microscope. The second image was captured in the same way after $48 \mathrm{~h}$. The closure of wound width in images was measured three times by Photoshop software. The wound healing rate $=$ (average wound width at $0 \mathrm{~h}$ ) - (average wound width at $48 \mathrm{~h}$ )/(average wound width at $0 \mathrm{~h}$ ) $\mathrm{x} 100 \%$.

Transfected cells were plated in Transwell cell culture inserts (Corning Costar, Corning, NY, USA) at a density of $2 \times 10^{4}$ cells/well. The cells were maintained and allowed to migrate for $48 \mathrm{~h}$, after which the cells that had not invaded were removed from the upper surface using a cotton swab. The cells that had invaded to the lower surface were stained with crystal violet solution and photographed under a microscope.

Glycolysis-related assays. Glucose Assay kit (ab65333), Deproteinizing Sample Preparation kit-TCA (ab204708), Glucose Uptake Assay kit (ab136955) and L-lactate assay kit (ab65330; all from Abcam, Cambridge, MA, USA) were 
A

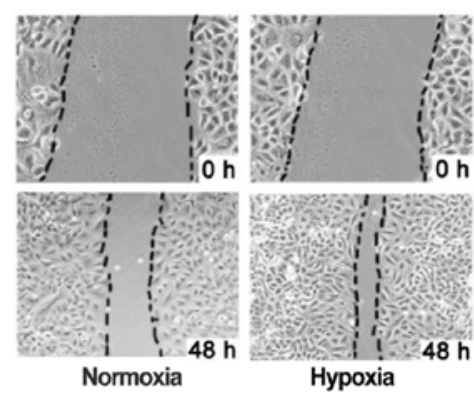

B

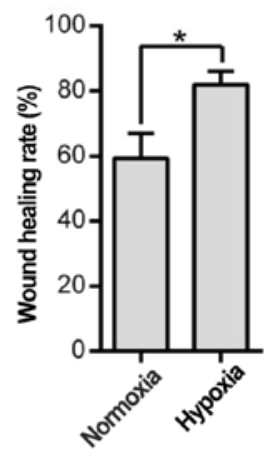

C

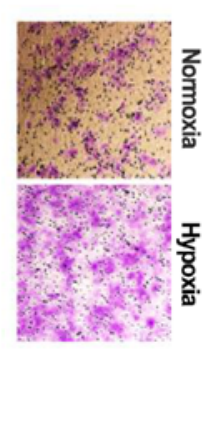

D

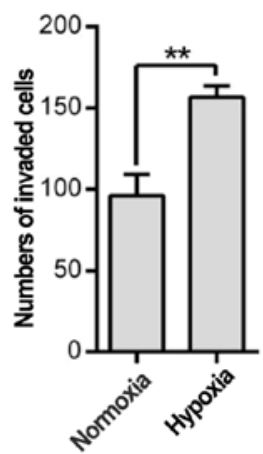

E

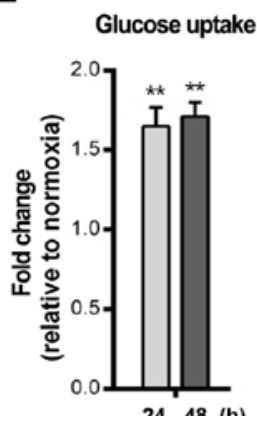

F

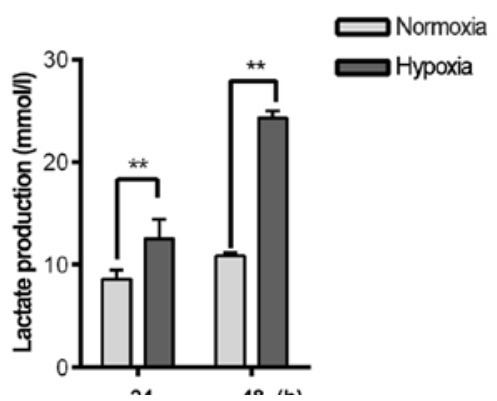

G

Figure 1. Hypoxia induces HNSCC metastasis and glycolysis in the Tu686 cell line. (A) Wound healing experiment showed that there was a different rate of migration between normoxic and hypoxic cells. (B) Quantitative analysis revealed that the percentage of wound healing in hypoxic Tu686 cells was significantly higher than that in the normoxic Tu686 cells $\left(82.0 \pm 4.0\right.$ vs. $59.3 \pm 7.6 \%$; $\left.{ }^{*} \mathrm{P}<0.05\right)$. (C) Representative figures for evaluation of cell invasion in hypoxic and normoxic Tu686 cells. (D) Quantitative analysis showed that hypoxia induced Tu686 cell invasion $\left(157 \pm 7\right.$ vs. 96 $\left.\pm 13 ;{ }^{* *} \mathrm{P}<0.01\right)$. (E and F) Glucose uptake and lactate production were significantly promoted in the hypoxic Tu686 cells after 24 and $48 \mathrm{~h} ;{ }^{* * *} \mathrm{P}<0.01$. (G) Amount of glucose in the supernatant was decreased in hypoxic Tu686 cells after 24 and $48 \mathrm{~h} ;{ }^{* *} \mathrm{P}<0.01$..

utilized in the glycolysis-related assays according to the manufacturer's protocols.

Statistical analysis. All statistical analyses were performed using IBM SPSS statistical software, version 21.0 (SPSS, Inc., Chicago, IL, USA). The differences between data derived from two groups in the experiments were statistically analyzed by a Student's test. $\mathrm{P}<0.05$ was considered to indicate a statistical significant result. All tests were two-sided.

\section{Results}

Hypoxia induces migration/invasion and glycolysis in a HNSCC cell line. Hypoxia is a hallmark of cancer cells. Thus, we investigated whether hypoxia induces cell migration and invasion in the HNSCC Tu686 cell line. The wound-healing rate indicated that hypoxia significantly promoted Tu686 cell healing after $48 \mathrm{~h}$ in hypoxia compared to cells cultured in normoxia $(\mathrm{P}<0.05$; Fig. $1 \mathrm{~A}$ and $\mathrm{B})$. A similar trend was observed in the Transwell experiment ( $\mathrm{P}<0.01$; Fig. $1 \mathrm{C}$ and $\mathrm{D})$. Considering that glycolysis is a fundamental stress-adaption process in hypoxic cells, glycolysis was analyzed in HNSCC cell lines at different time points. Glucose uptake and lactate secretion were increased in the Tu686 cells after 24 and $48 \mathrm{~h}$ of hypoxia treatment $(\mathrm{P}<0.01$; Fig. $1 \mathrm{E}$ and $\mathrm{F})$. In addition, the concentration of glucose in the cell culture supernatant was reduced significantly $(\mathrm{P}<0.01$; Fig. $1 \mathrm{G})$. Furthermore, several glycolytic genes, including MCT1, GLUT1 and PGI, were overexpressed in the hypoxic cells (data not shown).
A
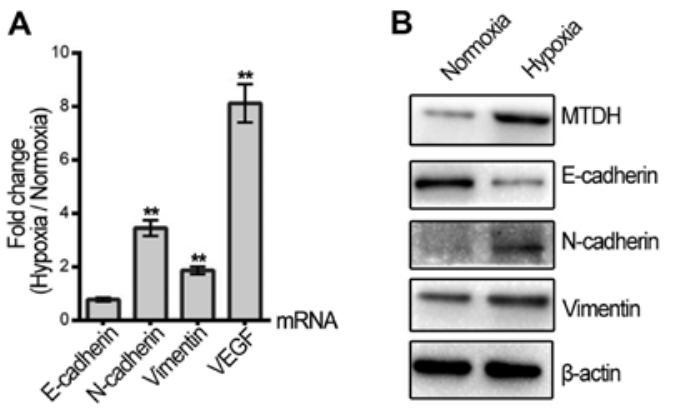

Figure 2. Hypoxia induces EMT and MTDH expression in HNSCC cells (A) mRNA expression of E-cadherin, N-cadherin, vimentin and VEGF was compared in hypoxic and normoxic Tu686 cells $\left({ }^{* *} \mathrm{P}<0.01\right)$. (B) Hypoxia induced MTDH protein expression and EMT. $\beta$-actin was used as an internal reference.

\section{Hypoxia promotes MTDH expression and induces EMT in an} HNSCC cell line. Since the migration and invasion of HNSCC cells were increased by hypoxic treatment, qPCR and western blotting were applied to assess whether hypoxia regulates the expression of VEGF and EMT biomarkers, which are key molecules involved in cell metastasis. VEGF mRNA was promoted in the hypoxic HNSCC cells. The mRNA and protein expression levels of vimentin and $\mathrm{N}$-cadherin were upregulated, while E-cadherin protein was decreased following hypoxic treatment (Fig. 2A and B). Furthermore, MTDH expression was significantly elevated in the Tu686 cells after $48 \mathrm{~h}$ of hypoxic treatment compared with normoxia (Fig. 2B). 
A

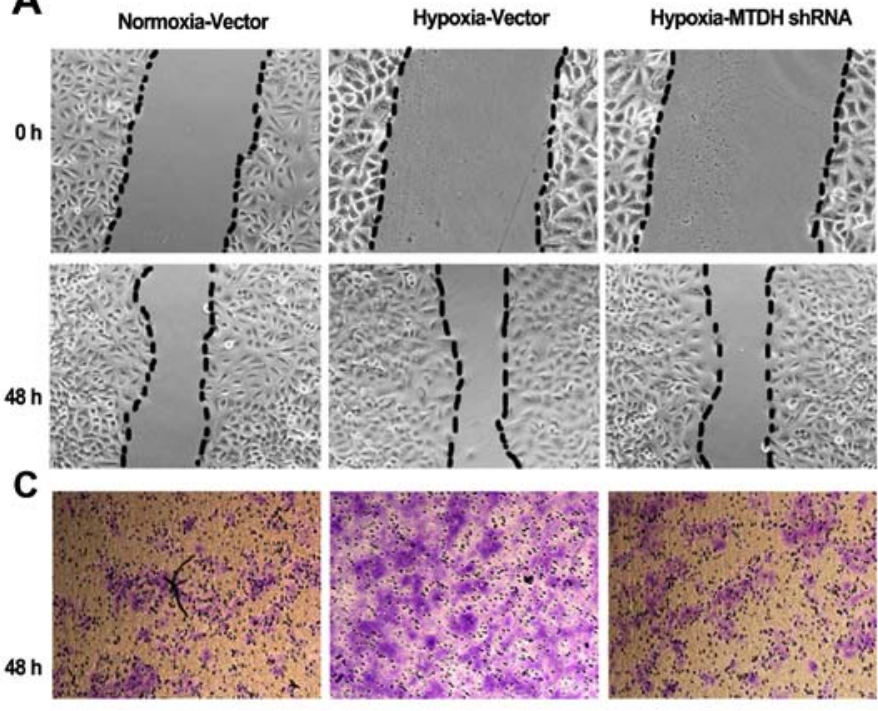

E

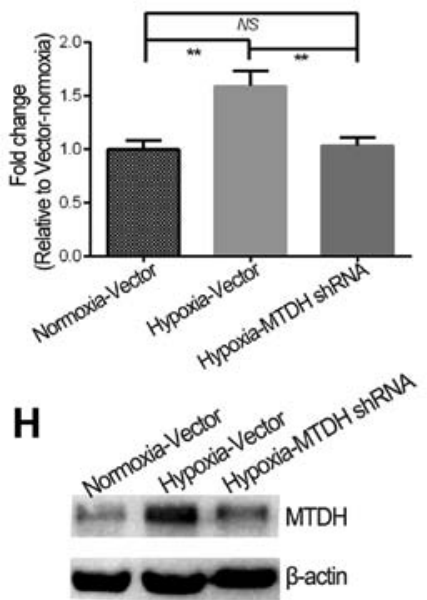

$\mathbf{F}$

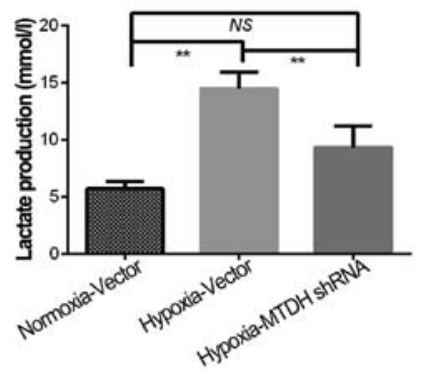

B

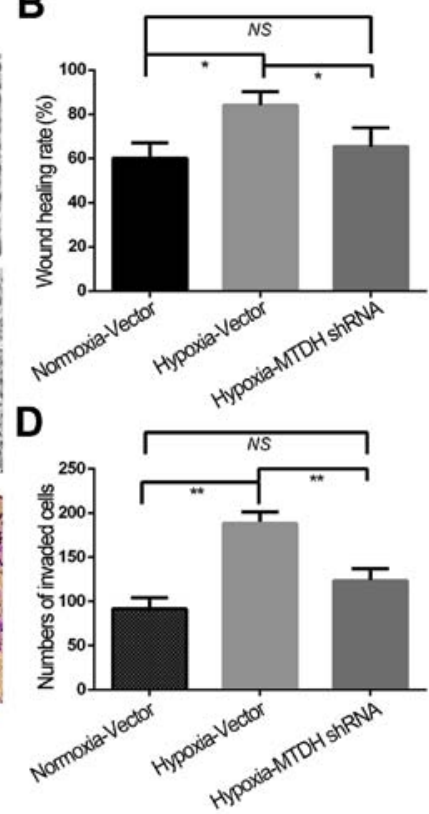

G

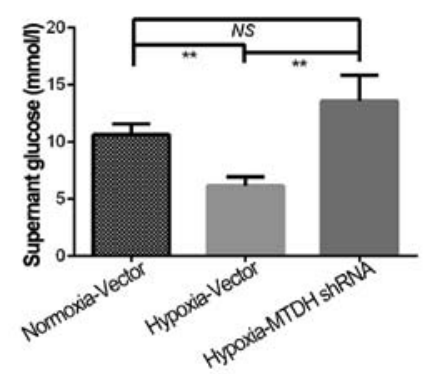

Figure 3. MTDH reverses HNSCC metastasis and glycolysis induced by hypoxia in the Tu686 cell line. (A) The percentage of wound closure in hypoxic cells with MTDH knockdown was approximately equal to that noted in normoxic cells. (B) Quantitative analysis revealed there was no significant difference in wound closure percentage in normoxic Tu686 and hypoxic Tu686 cells with MTDH knockdown (60.0 \pm 7.0 vs. $65.3 \pm 8.5 \%$, respectively; NS indicates $\mathrm{P}>0.05)$. Meanwhile, the percentage of wound closure in hypoxic cells was significantly higher than both of normoxic cells and hypoxic cells with MTDH downregulation $\left(84.0 \pm 6.2\right.$ vs. $60.0 \pm 7.0$ and $65.3 \pm 8.5 \%$, respectively; $\left.{ }^{*} \mathrm{P}<0.05\right)$. (C) The number of invading cells in hypoxic cells with MTDH knockdown was approximately equal to the number of normoxic cells. (D) There was no significant difference in invading cell number in normoxic Tu686 and hypoxic Tu686 cells with MTDH knockdown $(92 \pm 13$ vs. $123 \pm 14$ respectively; NS indicates $P>0.05)$. The invading cell number in hypoxic cells was significantly higher than both of normoxic cells and hypoxic cells with MDH downregulation (188 \pm 13 vs. $92 \pm 13$ and $123 \pm 14$, respectively; $\left.{ }^{* *} \mathrm{P}<0.01\right)$. (E and F) The promotion of glucose uptake and lactate production by hypoxia were significantly reversed by knockdown of MTDH expression. (G) The amount of glucose in the supernatant of hypoxic cells was increased to the level of nomoxic cells. (H) Western blotting showed that the MTDH protein was significantly decreased in the cells with MTDH shRNA transfection.

Hypoxia induces HNSCC cell metastasis and glycolysis by regulation of $M T D H$. High expression of MTDH protein was previously shown to predict poor prognosis in laryngeal squamous cell carcinoma patients (12). Although MTDH expression and migration/invasion were increased in HNSCC cells treated with hypoxia, the role of MTDH in hypoxia-regulated HNSCC metastasis remained to be uncovered. Therefore, MTDH expression was knocked down in the HNSCC cells (Fig. 3H) and further experiments were performed. The wound healing and invasion experiments indicated that knockdown of MTDH expression in hypoxic cells significantly reduced cell migration and invasion to the levels observed in normoxia (Fig. 3A-D).
Additionally, glycolysis was blocked in hypoxic cells when MTDH expression was knocked down $(\mathrm{P}<0.01$; Fig. 3E and G). Furthermore, lactate production in hypoxic cells with MTDH knockdown was almost reduced to the normoxic level (Fig. 3F).

Mutual regulation of MTDH and HIF-1 $\alpha$ in an HNSCC cell line. As the aforementioned results indicated that hypoxia regulated MTDH expression, we subsequently investigated whether HIF-1 $\alpha$, a classic indicator of hypoxia, regulated MTDH expression in Tu686 cells. MTDH expression was assessed in cells transfected with HIF-1 $\alpha$ cDNA and siRNA. 


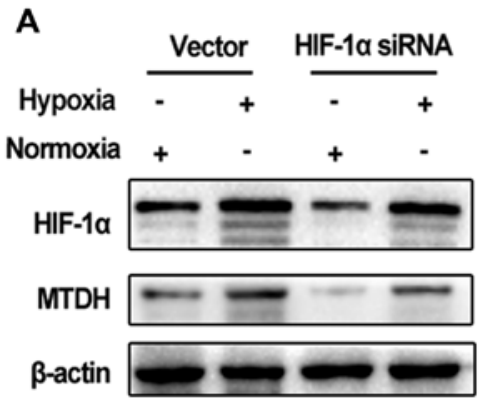

C

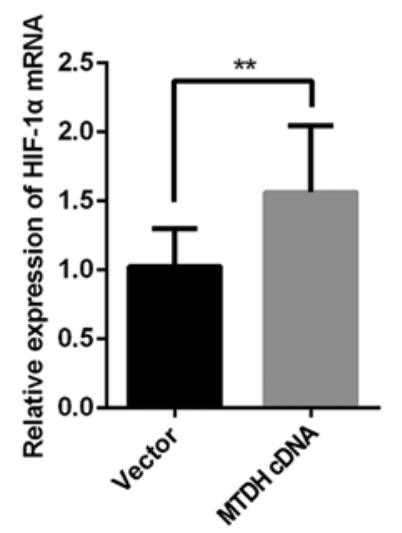

B

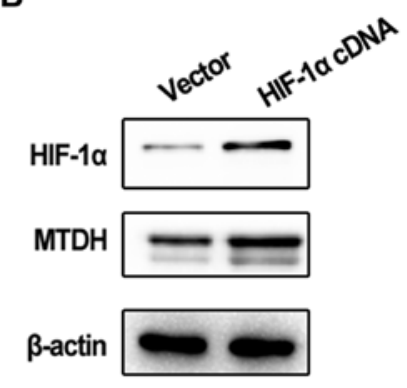

D

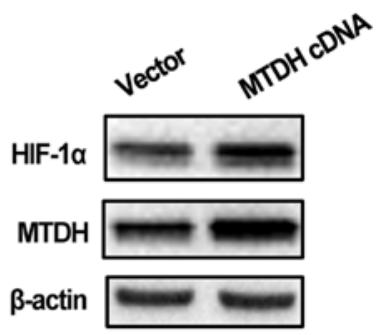

Figure 4. Mutual regulation of HIF-1 $\alpha$ and MTDH in Tu686 cells. (A) MTDH protein expression was decreased when HIF-1 $\alpha$ siRNA was transfected in both normoxic and hypoxic cells. (B) Compared to Tu686 cells transfected with the plasmid vector, MTDH protein expression was upregulated in Tu686 cells with HIF-1 $\alpha$ cDNA transfection. (C) HIF-1 $\alpha$ mRNA was significantly upregulated in MTDH-overexpressing cells ( $(*$ P $<0.01)$. (D) Western blotting indicated that MTDH protein was significantly increased in the cells transfected with MTDH cDNA. In addition, HIF-1 $\alpha$ protein was promoted in the MTDH-overexpressing cells.

A

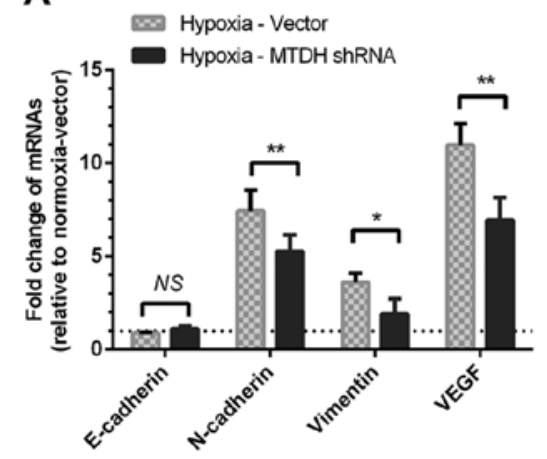

B

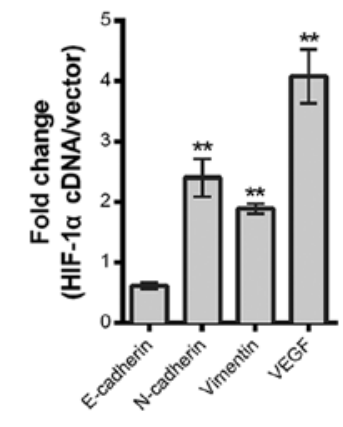

C

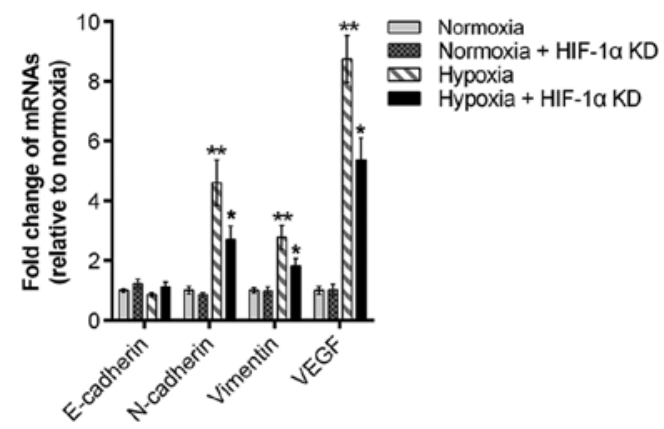

Figure 5. MTDH and HIF-1 $\alpha$ regulate EMT-related biomarkers and VEGF expression in Tu686 cells. (A) mRNA expression of E-cadherin, N-cadherin, vimentin and VEGF was compared in normoxic Tu686, hypoxic Tu686 and hypoxic Tu686 cells with MTDH knockdown ("P $<0.05$, ** P $<0.01)$. (B) HIF-1 $\alpha$ induced the expression of $\mathrm{N}$-cadherin, vimentin and VEGF mRNA $\left({ }^{* *} \mathrm{P}<0.01\right)$. (C) The expression of $\mathrm{N}$-cadherin, vimentin and VEGF mRNA were decreased by HIF-1 $\alpha$ knockdown in normoxic and hypoxic Tu686 cells $\left({ }^{*} \mathrm{P}<0.05,{ }^{* *} \mathrm{P}<0.01\right)$. NS indicates $\mathrm{P}>0.05$.

The expression level of MTDH protein was markedly decreased when HIF-1 $\alpha$ was knocked down in the Tu686 cell line under hypoxic and normoxic conditions (Fig. 4A). Furthermore, MTDH expression in hypoxic cells with HIF-1 $\alpha$ knockdown was reduced to a similar level as that observed in cells cultured under normoxia (lane 1 vs. 4; Fig. 4A), suggesting that the hypoxia-induced increase in the expression of MTDH in Tu686 cells could be attenuated by HIF-1 $\alpha$ knockdown. In addition, upregulation of HIF-1 $\alpha$ significantly increased MTDH protein expression (Fig. 4B). Notably, the expression of
HIF-1 $\alpha$ mRNA was increased significantly in HNSCC Tu686 cells in which MTDH was overexpressed (Fig. 4C and D).

MTDH or HIF-1a knockdown partially reverses the hypoxia-induced promotion of EMT and VEGF expression in HNSCC cells. Our previous studies showed that MTDH regulated HNSCC metastasis by altering the expression of EMT proteins and VEGF in normoxic cell culture $(13,14)$. In the present study, compared with the plasmid vector group, downregulation of MTDH decreases the mRNA expression 
levels of vimentin, VEGF, and N-cadherin when cells were cultured under hypoxic conditions $(\mathrm{P}<0.01$; Fig. $5 \mathrm{~A})$. Considering the MTDH and HIF-1 $\alpha$ loop in HNSCC, whether HIF-1 $\alpha$ regulated these EMT and angiogenesis biomarkers was assessed. The mRNA levels of vimentin, $\mathrm{N}$-cadherin and VEGF were upregulated by HIF- $1 \alpha$ cDNA overexpression (Fig. 5B). Meanwhile, decreased expression levels of vimentin, $\mathrm{N}$-cadherin and VEGF mRNA were detected when HNSCC cells were transfected with HIF-1 $\alpha$ siRNA (Fig. 5C).

\section{Discussion}

In the present study, we confirmed that hypoxia induced cell metastasis, glycolysis and MTDH expression in an HNSCC cell line. Additionally, downregulation of MTDH expression could offset the hypoxia-induced increase in HNSCC cell metastasis and glycolysis. We also verified that HIF-1 $\alpha$ was crucial for the regulation of MTDH by hypoxia. Importantly, MTDH was found to regulate HIF-1 $\alpha$ expression in turn. Furthermore, both MTDH and HIF- $1 \alpha$ could partially reverse the hypoxia-induced increases in EMT biomarkers and VEGF expression in HNSCC cells.

A range of cellular signaling pathways, relating to angiogenesis, autophagy, EMT and energy metabolism, are activated in cells in order to adapt to hypoxic stress (17). Hypoxia is a hallmark of solid cancers and promotes cell metastasis in several ways. The present study indicated that hypoxic treatment of the Tu686 HNSCC cell line induced migration and invasion in these cells, which is consistent with the findings of previous studies in other cell lines (18). However, the detailed mechanism underlying hypoxia-induced cell metastasis remains unclear. HIF- $1 \alpha$ is considered a representative biomarker of hypoxia, and glycolysis is also associated with the hypoxic process. Therefore, hypoxia may promote cell metastasis through HIF-1 $\alpha$-mediated transcriptional changes and glycolysis-mediated energy changes.

When cells are cultured in an environment of $1 \% \mathrm{O}_{2}, \mathrm{HIF}-1 \alpha$ protein is stabilized by a reduction in ubiquitination-mediated degradation (19). The present study confirmed that HIF-1 $\alpha$ protein, rather than mRNA, was increased in hypoxic HNSCC cells (data not shown). HIF-1 $\alpha$, which is a sensitive indicator of hypoxia, is translocated to the nucleus to regulate transcription. Numerous target gene transcripts, including MMPs, EMT biomarkers and VEGF, are increased in hypoxic microenvironments since they contain hypoxia-response elements(HRE) (6).

The present study showed that hypoxia increased the mRNA expression levels of glycolysis-related genes, such as MCT1, MCT4, GLUT1, HK2, PGI, PGK1, ENO1, PFK2 and LDHA, in HNSCC cells (data not shown). In addition, our data showed that hypoxia promoted glucose uptake, lactate production and cell invasion in HNSCC cells. In the clinical setting, glucose uptake has been used in PET-CT imaging for assessing tumor metastasis and relapse (20). Lactate was recently investigated as a predictive biomarker for tumor recurrence and poor survival time based on data from HNSCC obtained over a period of 15 years (21). High level of the lactate generator LDHA is an indicator of poor prognosis in HNSCC patients (22). Glycolytic enzymes, such as HK2, PGI, PGK1,
ENO1 and PFK2, are involved in cancer anaerobic glycolysis and metastasis (23). Elevated expression levels of MCT1/4, a protein responsible for lactate shuttle, were detected in breast and cervical cancer $(24,25)$. Genetic depletion of MCT1/4 in breast cancer impairs cell migration $(26,27)$, which may partially mediate the effect of glycolysis on HNSCC metastasis.

Notably, the present study indicated that MTDH protein expression was elevated in hypoxic HNSCC cells. MTDH is well-documented as an oncoprotein in human malignancies, including lung, colon, breast, live, glioma cancer and HNSCC $(12,28)$. Dysregulated MTDH expression levels in cancer may mediate tumor proliferation, progression and sensitivity to chemoradiation (11). Our previous data showed that MTDH is an unfavorable factor for HNSCC patient survival time, and promotes HNSCC cell metastasis and angiogenesis (12-14). Therefore, whether MTDH is involved in hypoxia-induced metastasis was studied. Knockdown of MTDH expression reversed the increase in cell migration and invasion in hypoxic HNSCC cells. Considering that hypoxia upregulates MTDH expression, this may indicate that hypoxia-promoted HNSCC cell metastasis partially depends on MTDH expression. Furthermore, the present study investigated the regulation of MTDH in HNSCC cells under hypoxic conditions with or without HIF-1 $\alpha$ mediation. This demonstrated that the induction of MTDH in HNSCC depends on HIF-1 $\alpha$ protein expression, which is consistent with a previous study in glioblastoma (15). The mechanism by which HIF-1 $\alpha$ regulates MTDH is not clear yet, although there are two hypotheses to explain this regulation. The first is that, since MTDH contains three HRE sites in its MTDH promoter (as identified through the web tool), HIF-1 $\alpha$ may promote MTDH transcription directly. The second is that HIF-1 $\alpha$ may increase MTDH expression indirectly via activation of c-Myc transcription $(29,30)$.

The multifaceted roles of MTDH in cancer are due to the diverse downstream cellular signals activated, including the PI3K/AKT, NF- $\mathrm{B}, \mathrm{Wnt} / \beta$-catenin and MAPK pathways (31). Emerging reports have indicated that genomic amplification (8q22 gain), transcriptional regulation (Ha-Ras/PI3K/c-Myc), and post-transcriptional and translational regulation (miRNA, CPEB1, Hbx and mono-ubiquitination) are involved in the mechanism of MTDH regulation in cancer (11). Furthermore, PI3K activation is crucial for protecting HIF-1 $\alpha$ from degradation (32). Considering that elevated MTDH expression has been shown to increase HNSCC cell metastasis through activating the PI3K/AKT pathway (13), whether MTDH regulated HIF-1 $\alpha$ in HNSCC remained unclear. The present study indicated that knockdown of MTDH in hypoxic HNSCC cells reduced cell invasion and migration to a level similar to that of normoxia-cultured HNSCC cells, implying that MTDH plays a vital role in hypoxic cell progression. Our results also indicated that MTDH regulated HIF-1 $\alpha$ expression in HNSCC. When cells are subjected to hypoxic stress, HIF-1 $\alpha$ may be activated to allow adaption to a different mode of energy generation by inducing the transcription of glycolytic genes. The glucose uptake and lactate production assays revealed that glycolysis was significantly decreased in hypoxic cells when MTDH was downregulated. Thus, the present study indicated that MTDH may regulate both HIF- $1 \alpha$ and HIF- $1 \alpha$-induced glycolysis in HNSCC cells with or without hypoxic stress. 
Our unpublished data showed that the NF- $\mathrm{BB}$ pathway is also involved in MTDH-induced mRNA/protein expression changes and cell metastasis. Given that HIF-1 $\alpha$ is one of the

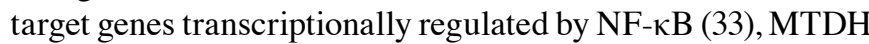
may also regulate HIF- $1 \alpha$ via the $N F-\kappa B$ pathway in HNSCC. The possible involvement of NF- $\kappa \mathrm{B}$ in MTDH regulation of $\mathrm{HIF}-1 \alpha$ requires further studies to confirm.

The mutual regulation of MTDH and HIF-1 $\alpha$ identified in the present study may indicate a feedback loop connection between MTDH and HIF-1 $\alpha$ in HNSCC. Additionally, it is possible that the intermodulation of MTDH and HIF-1 $\alpha$ may affect cell metastasis and EMT together. The present investigation indicated that EMT biomarkers and cell metastasis were enhanced significantly in hypoxic HNSCC cells. When MTDH shRNA was transfected into hypoxic cells (and HIF-1 $\alpha$ was also activated), EMT biomarker expression and cell metastasis were reduced, suggesting that disruption of the MTDH-HIF-1 $\alpha$ loop connection inhibited HNSCC cell metastasis and increased its resistance to EMT. There are two possible mechanisms: HIF-1 $\alpha$ may activate the EMT transcription factor Twist in hypoxic HNSCC cells (34), or MTDH may mediate the epigenetic regulation of Twist in cancer cells (35). There may be multiple links between MTDH, HIF-1 $\alpha$ and Twist in HNSCC. The current results indicated that decreased MTDH reversed the promotion of cell metastasis by hypoxia, which may imply that hypoxia-regulated HNSCC cell metastasis depends on MTDH expression. However, downregulation of MTDH and HIF-1 $\alpha$ partially reversed the induction of EMT biomarkers by hypoxia, which may indicate that hypoxia promoted HNSCC EMT via a complex pathway of mediators other than MTDH and HIF-1 $\alpha$.

Considering the aforementioned findings in HNSCC, there may be a loop connection between MTDH and HIF-1 $\alpha$ in HNSCC cells, and hypoxia may promote HNSCC cell glycolysis and metastasis via this loop. If cells grow at a sufficient distance from the oxygen-delivering vasculature within HNSCC tumors, adaption to hypoxic stress occurs, resulting in the nuclear translocation of HIF-1 $\alpha$ to promote the transcription of glycolytic genes and oncogenes, including LDHA and $\mathrm{MTDH}$, to accelerate energy synthesis and progression, respectively. MTDH activates the PI $3 \mathrm{~K}$ and $\mathrm{NF}-\kappa \mathrm{B}$ pathways, which may increase HIF-1 $\alpha$ expression in turn and further enhance the aforementioned process. The present study also found that HNSCC cell migration/invasion and glycolysis in hypoxia were suppressed when this positive feedback was disrupted by knockdown of MTDH expression. Thus, targeting MTDH in HNSCC may be a promising direction for improving the survival time of HNSCC patients, as MTDH reduced the malignant activity of HNSCC cells in normoxia and hypoxia. In summary, hypoxia promotes HNSCC cell migration/invasion and glycolysis by mediating a HIF-1 $\alpha-\mathrm{MTDH}$ feedback loop. These findings implicate HIF-1 $\alpha$-MTDH as a promising target for anticancer drugs in solid tumors, and help to explain the pro-tumorigenic and unfavorable effect of MTDH in HNSCC as reported in our previous study.

\section{Acknowledgements}

We thank all our laboratory members for their insightful suggestions in discussion. The present study was funded by grants from the National Natural Science Foundation of China (nos. 81602389, 81472696, 81202128 and 81272974), and the Natural Science Foundation of Hunan Province (nos. 2017JJ3456 and 2015JJ3137).

\section{References}

1. Torre LA, Bray F, Siegel RL, Ferlay J, Lortet-Tieulent J and Jemal A: Global cancer statistics, 2012. CA Cancer J Clin 65: 87-108, 2015.

2. Mamelle G, Pampurik J, Luboinski B, Lancar R, Lusinchi A and Bosq J: Lymph node prognostic factors in head and neck squamous cell carcinomas. Am J Surg 168: 494-498, 1994.

3. Quail DF and Joyce JA: Microenvironmental regulation of tumor progression and metastasis. Nat Med 19: 1423-1437, 2013

4. Vaupel P and Mayer A: Hypoxia in cancer: Significance and impact on clinical outcome. Cancer Metastasis Rev 26: 225-239, 2007.

5. Rankin EB and Giaccia AJ: Hypoxic control of metastasis. Science 352: 175-180, 2016

6. Palazon A, Goldrath AW, Nizet V and Johnson RS: HIF transcription factors, inflammation, and immunity. Immunity 41: 518-528, 2014.

7. Liu W, Shen SM, Zhao XY and Chen GQ: Targeted genes and interacting proteins of hypoxia inducible factor-1. Int J Biochem Mol Biol 3: 165-178, 2012.

8. Kim JW, Tchernyshyov I, Semenza GL and Dang CV: HIF-1mediated expression of pyruvate dehydrogenase kinase: A metabolic switch required for cellular adaptation to hypoxia. Cell Metab 3: 177-185, 2006.

9. Lu J, Tan M and Cai Q: The Warburg effect in tumor progression: Mitochondrial oxidative metabolism as an anti-metastasis mechanism. Cancer Lett 356: 156-164, 2015.

10. Verdone JE, Zarif JC and Pienta KJ: Aerobic glycolysis, motility, and cytoskeletal remodeling. Cell Cycle 14: 169-170, 2015.

11. Emdad L, Das SK, Hu B, Kegelman T, Kang DC, Lee SG, Sarkar D and Fisher PB: AEG-1/MTDH/LYRIC: A promiscuous protein partner critical in cancer, obesity, and CNS diseases. Adv Cancer Res 131: 97-132, 2016.

12. Liu Y, Su Z, Li G, Yu C, Ren S, Huang D, Fan S, Tian Y, Zhang X and Qiu Y: Increased expression of metadherin protein predicts worse disease-free and overall survival in laryngeal squamous cell carcinoma. Int J Cancer 133: 671-679, 2013.

13. Yu C, Liu Y, Tan H, Li G, Su Z, Ren S, Zhu G, Tian Y, Qiu Y and Zhang X: Metadherin regulates metastasis of squamous cell carcinoma of the head and neck via AKT signalling pathwaymediated epithelial-mesenchymal transition. Cancer Lett 343: 258-267, 2014.

14. Zhu GC, Yu CY, She L, Tan HL, Li G, Ren SL, Su ZW, Wei M, Huang DH, Tian YQ, et al: Metadherin regulation of vascular endothelial growth factor expression is dependent upon the PI3K/Akt pathway in squamous cell carcinoma of the head and neck. Medicine 94: e502, 2015.

15. Noch E, Bookland M and Khalili K: Astrocyte-elevated gene-1 (AEG-1) induction by hypoxia and glucose deprivation in glioblastoma. Cancer Biol Ther 11: 32-39, 2011.

16. Zhu G, Cai G, Liu Y, Tan H, Yu C, Huang M, Wei M, Li S, Cui X, Huang D, et al: Quantitative iTRAQ LC-MS/MS proteomics reveals transcription factor crosstalk and regulatory networks in hypopharyngeal squamous cell carcinoma. J Cancer 5: 525-536, 2014.

17. Pouysségur J, Dayan F and Mazure NM: Hypoxia signalling in cancer and approaches to enforce tumour regression. Nature 441: 437-443, 2006.

18. Shan Y, Li X, You B, Shi S, Zhang Q and You Y: MicroRNA-338 inhibits migration and proliferation by targeting hypoxia-induced factor 1 $\alpha$ in nasopharyngeal carcinoma. Oncol Rep 34: 1943-1952, 2015.

19. Yee Koh M, Spivak-Kroizman TR and Powis G: HIF-1 regulation: Not so easy come, easy go. Trends Biochem Sci 33: 526-534, 2008.

20. Gupta T, Master Z, Kannan S, Agarwal JP, Ghsoh-Laskar S, Rangarajan V, Murthy V and Budrukkar A: Diagnostic performance of post-treatment FDG PET or FDG PET/CT imaging in head and neck cancer: A systematic review and meta-analysis. Eur J Nucl Med Mol Imaging 38: 2083-2095, 2011. 
21. Blatt S, Voelxen N, Sagheb K, Pabst AM, Walenta S, Schroeder T, Mueller-Klieser W and Ziebart T: Lactate as a predictive marker for tumor recurrence in patients with head and neck squamous cell carcinoma (HNSCC) post radiation: A prospective study over 15 years. Clin Oral Investig 20: 2097-2104, 2016.

22. Koukourakis MI, Giatromanolaki A, Winter S, Leek R, Sivridis E and Harris AL: Lactate dehydrogenase 5 expression in squamous cell head and neck cancer relates to prognosis following radical or postoperative radiotherapy. Oncology 77: 285-292, 2009.

23. Payen VL, Porporato PE, Baselet B and Sonveaux P: Metabolic changes associated with tumor metastasis, part 1: Tumor $\mathrm{pH}$, glycolysis and the pentose phosphate pathway. Cell Mol Life Sci 73: 1333-1348, 2016.

24. Doherty JR and Cleveland JL: Targeting lactate metabolism for cancer therapeutics. J Clin Invest 123: 3685-3692, 2013.

25. Pinheiro C, Longatto-Filho A, Ferreira L, Pereira SM, Etlinger D, Moreira MA, Jubé LF, Queiroz GS, Schmitt F and Baltazar F: Increasing expression of monocarboxylate transporters 1 and 4 along progression to invasive cervical carcinoma. Int J Gynecol Pathol 27: 568-574, 2008.

26. Levenson AS, Thurn KE, Simons LA, Veliceasa D, Jarrett J, Osipo C, Jordan VC, Volpert OV, Satcher RL Jr and Gartenhaus RB: MCT-1 oncogene contributes to increased in vivo tumorigenicity of MCF7 cells by promotion of angiogenesis and inhibition of apoptosis. Cancer Res 65: 10651-10656, 2005.

27. Gallagher SM, Castorino JJ, Wang D and Philp NJ: Monocarboxylate transporter 4 regulates maturation and trafficking of CD147 to the plasma membrane in the metastatic breast cancer cell line MDA-MB-231. Cancer Res 67: 4182-4189, 2007.

28. Lee S-G, Kang D-C, DeSalle R, Sarkar D and Fisher PB: AEG-1/MTDH/LYRIC, the beginning: Initial cloning, structure, expression profile, and regulation of expression. Adv Cancer Res 120: 1-38, 2013.
29. Podar $\mathrm{K}$ and Anderson KC: A therapeutic role for targeting c-Myc/Hif-1-dependent signaling pathways. Cell Cycle 9: $1722-1728,2010$.

30. Lee SG, Su ZZ, Emdad L, Sarkar D and Fisher PB: Astrocyte elevated gene-1 $(A E G-1)$ is a target gene of oncogenic Ha-ras requiring phosphatidylinositol 3-kinase and c-Myc. Proc Natl Acad Sci USA 103: 17390-17395, 2006.

31. Emdad L, Das SK, Dasgupta S, Hu B, Sarkar D and Fisher PB: AEG-1/MTDH/LYRIC: Signaling pathways, downstream genes, interacting proteins, and regulation of tumor angiogenesis. Adv Cancer Res 120: 75-111, 2013.

32. Mottet D, Dumont V, Deccache Y, Demazy C, Ninane N, Raes M and Michiels C: Regulation of hypoxia-inducible factor-1alpha protein level during hypoxic conditions by the phosphatidylinositol 3-kinase/Akt/glycogen synthase kinase 3beta pathway in HepG2 cells. J Biol Chem 278: 31277-31285, 2003.

33. Rius J, Guma M, Schachtrup C, Akassoglou K, Zinkernagel AS, Nizet V, Johnson RS, Haddad GG and Karin M: NF-kappaB links innate immunity to the hypoxic response through transcriptional regulation of HIF-1alpha. Nature 453: 807-811, 2008.

34. Yang MH, Wu MZ, Chiou SH, Chen PM, Chang SY, Liu CJ, Teng SC and Wu KJ: Direct regulation of TWIST by HIF-1alpha promotes metastasis. Nat Cell Biol 10: 295-305, 2008.

35. Liang Y, Hu J, Li J, Liu Y, Yu J, Zhuang X, Mu L, Kong X, Hong D, Yang Q, et al: Epigenetic activation of TWIST1 by MTDH promotes cancer stem-like cell traits in breast cancer. Cancer Res 75: 3672-3680, 2015. 